\title{
Use of the polymerase chain reaction in the diagnosis of leprosy
}

\author{
A. R. SANTOS, J. C. NERY, NADIA C. DUPPRE, MARIA E. N. GALLO, J. T. G. FILHO*, \\ P. N. SUFFYS* and W. M. DEGRAVE*
}

Department of Tropical Medicine, Leprosy Sector and *Department of Biochemistry and Molecular Biology, Oswaldo Cruz Institute, Fiocruz, Av. Brasil 4365, Manguinhos 21045-900, Rio de Janeiro, RJ, Brazil

\begin{abstract}
One of the main limitations for successful epidemiological control of leprosy is the lack of a method for its diagnosis in subclinical cases. Because of the long incubation period of the disease, liberation and spread of Mycobacterium leprae during subclinical stages principally in cases of untreated multibacillary forms of leprosy - constitute the main source of infection. This report describes the use of the polymerase chain reaction (PCR) for the detection of $M$. leprae in different types of tissue samples (blood, lymph, nasal secretion and hair) from an individual who was suspected of having leprosy. Although no conclusive diagnosis could be made by traditional diagnostic methods, the individual was found to be infected with $M$. leprae after amplification of the bacterial DNA.
\end{abstract}

\section{Introduction}

Leprosy is an infectious disease of man and occurs principally in developing countries. Its global prevalence, estimated to be 5.5 million in 1991, has decreased dramatically over the last decade because of the introduction of multidrug therapy. Nevertheless, leprosy still represents one of the major public health problems in many countries of Africa and Latin America.

Disease transmission is maintained mainly by the spread of bacilli from advanced and untreated multibacillary cases as well as by the untreated multibacillary forms in a subclinical stage of infection, for which, until now, diagnosis has proved impossible. Patients usually remain untreated until clinical symptoms appear or until acid-fast bacilli can be demonstrated in skin biopsy or lymph from the ear lobe.

In the last few years, several reports have suggested the use of the polymerase chain reaction (PCR) for diagnosis of subclinical leprosy [1-4]. As demonstrated earlier, DNA from Mycobacterium leprae, present in different kinds of clinical material such as lymph, blood, biopsy samples [4], as well as in nasal secretion and in hair bulbs [5], can be amplified and detected with a set of oligonucleotide primers complementary to part of an $M$. leprae-specific repetitive sequence [6]. To achieve this, PCR condi-

Received 12 March 1996; accepted 3 July 1996.

Corresponding author: Dr P. Suffys. tions for detection of $M$. leprae DNA in multi- and paucibacillary patients were optimised [4,5] and, considering the sensitivity of PCR, it was hoped that this method would serve as a suitable complement in cases with a doubtful diagnosis. This report describes the detection by PCR of $M$. leprae DNA in clinical specimens, from a patient with leprosy that was difficult to diagnose and discusses the potential of this technique for detection of infected individuals within a population at risk.

\section{Patient and methods}

A 32-year-old woman presented with pain and parasthesia in the ulnar region of the right forearm. Dermatological examination revealed a hypopigmented macule on the right forearm without loss of sensitivity, typical symptoms of leprosy. The woman was submitted to the following routine procedures for leprosy diagnosis: dermatological examination to detect other lesions; neurological examination including electroneuromyography; skin biopsy sampling for histopathological evaluation and determination of the bacteriological and morphological index by microscopy. A lepromin test was also performed.

No lepromin reaction was observed and the bacteriological index was found to be zero. The result of the electroneuromyography was also inconclusive. Neurological evaluation revealed a painful thickening of the ulnar nerve in the right hand and of the left tibial nerve in the leg. Screening for fungal parasites was 
performed on the lesion of the forearm and revealed the presence of hyphae compatible with Malassessia furfur. Haematoxylin and eosin-stained histological skin sections showed a small perivascular inflammatory infiltrate consisting of lymphocytes and histiocytes in the upper dermis. Periodic-acid-Schiff (PAS)positive structures corresponding to hyphae were found in the corneal layer of the epidermis.

Based on these results, the diagnosis at that time was dermatophytosis mycoses and specific chemotherapy was prescribed. One month later, the patient still presented with the same clinical signs, although the lesion had almost disappeared. The patient was submitted to the same clinical and laboratory protocol and again, no definitive leprosy diagnosis could be reached.

In an attempt to avoid a nerve biopsy, a highly invasive procedure, it was decided to test a PCR assay on different types of clinical samples from this patient. Two PCR assays were performed and for the first PCR, hair bulbs were picked from an area suspected of bacterial infiltration; blood and nasal secretions were also collected. One month later, a second PCR assay was performed on lymph from the left ear lobe and from the residual lesion, and, in addition, fresh samples of blood, nasal secretion and hair bulb were taken. Sample processing and PCR conditions were as described previously $[4,5]$ and amplified product samples were analysed by gel electrophoresis after ethidium bromide staining and by hybridisation with an oligonucleotide probe, internal to the amplified region [4]. To avoid false positive results, negative controls (without DNA) and normal controls (similar clinical samples from an individual without contact with $M$. leprae) were included in each test.

\section{Results and discussion}

PCR analyses were performed on clinical samples which had been collected with an interval of 1 month. During the first PCR, blood, nasal secretion and hair bulbs were analysed; of these only nasal secretions gave a positive signal. Based on earlier results [5] and on data from De Wit et al. [2,7], it was suspected that a positive PCR signal for $M$. leprae in nasal secretions does not necessarily mean that the individual under study is actually infected with the bacilli and especially in an endemic region of leprosy - can reflect passive carriage of bacteria. As the person under investigation was a nurse who had professional contact with leprosy patients, further samples (including lymph) were analysed 1 month after the first assay. The results of the second PCR analysis are shown in Fig. 1. Not only was the PCR positivity of the nasal secretions confirmed, but at that time, blood was also found to be positive both after visual (Fig. 1b) and hybridisation (Fig. 1d) analysis, thus confirming infection with $M$. leprae. At the time of diagnosis, the Brazilian Ministry of Health recommended that all patients with a negative lepromin reaction should be treated by the WHO multibacillary protocol, independent of the bacteriological index [8].

The patient was treated with 24 monthly supervised combined doses of rifampicin $600 \mathrm{mg}$ and clofazimine $300 \mathrm{mg}$, and daily self-administered doses of diaminediphenyl sulphone $100 \mathrm{mg}$ and clofazimine $50 \mathrm{mg}$, after which the patient was re-evaluated. The clinical signs presenting 2 years earlier had disappeared and the patient was therefore discharged from further follow-up.

Based on these results, we consider that the PCR technique could be used as a complementary approach

$\begin{array}{llllllllllllllllll}1 & 2 & 3 & 4 & 5 & 6 & 7 & 8 & 9 & 1 & 2 & 3 & 4 & 5 & 6 & 7 & 8 & 9\end{array}$

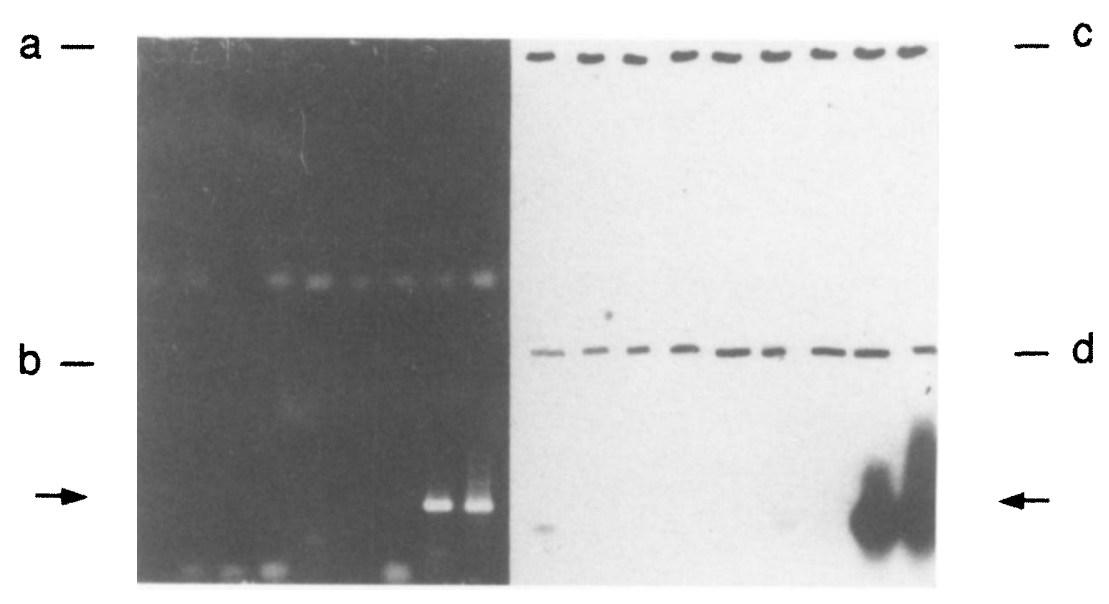

Fig. 1. PCR products obtained after amplification of $M$. leprae DNA in different clinical samples after the second PCR analysis, as shown by visual analysis after ethidium bromide staining $(\mathbf{a}+\mathbf{b})$ or by hybridisation analysis $(\mathbf{c}+\mathbf{d})$. $\mathbf{a}, \mathbf{c}$ : lanes 1, 2, lymph; 3, negative control; 4-7, hair bulbs; 8, normal control; 9, negative control. b, d: lanes 1, 2, nasal secretion; 3, normal control; 4, negative control; 5, 6, peripheral blood mononuclear cells; 7, negative control; 8, 9, positive control ( $M$. leprae DNA). Arrow indicates the amplified product with a mol. wt of $372 \mathrm{bp}$. 
in leprosy cases that are difficult to diagnose. Its use as an additional diagnostic tool during screening of at risk populations should be considered and could help in the interruption of the leprosy transmission chain.

This work received financial support from the World Health Organization (UNDP/WORLD BANK/WHO) Special Programme for Research and Training in Tropical Diseases (TDR) and Faperj. We thank the staff of the Souza Araujo Ambulatory for patient care and sample collection.

\section{References}

1. Gillis TP, Williams DL. Polymerase chain reaction and leprosy. Int J Lepr Other Mycobact Dis 1991; 59: 311-316.

2. De Wit MYL, Douglas JT, McFadden J, Klatser PR. Polymerase chain reaction for detection of Mycobacterium leprae in nasal swab specimens. J Clin Microbiol 1993; 31: 502-506.

3. Ayliffe PR. Modern sensitive techniques for the detection of Mycobacterium leprae. Int $J$ Lepr 1992; 60: 451-464.

4. Santos AR, De Miranda AB, Sarno EN, Suffys PN, Degrave WM. Use of PCR-mediated amplification of Mycobacterium leprae DNA in different types of clinical samples for the diagnosis of leprosy. J Med Microbiol 1993; 39: 298-304.

5. Santos AR, Goes-Filho JT, Nery JAC et al. Evaluation of PCR mediated DNA amplification in non-invasive biological specimens for subclinical detection of Mycobacterium leprae. FEMS Immunol. Microbiol. 1995; 11: 113-120.

6. Clark-Curtiss J, Docherty MA. A species-specific repetitive sequence in Mycobacterium leprae DNA. J Infect Dis 1989; 159: $7-15$.

7. Klatser PR, van Beers S, Madjid B, Day R, De Wit MYL. Detection of Mycobacterium leprae nasal carriers in populations for which leprosy is endemic. J Clin Microbiol 1993; 31: 2947-2951.

8. Ministry of Health, National Division of Sanitary Dermatology, Resolution no. 01, official diary no. 02/90, Section I, January, 1990. 110-114. Brasilia, DF, Brazil. 